\title{
Determinación de material y condiciones de trabajo del Torno CNC en la operación de cilindrado
}

Determination of materials and cutting conditions in a CNC lathe turning operation

Juan Bautista Hernández-Granados'

Marcela Meneses-Guzmán²

Federico Picado-Alvarado ${ }^{3}$

Fecha de recepción: 30 de julio del 2013

Fecha de aprobación: 02 de diciembre del 2013

Hernández-Granados, J; Meneses-Guzmán, M;

Picado-Alvarado, F. Determinación de material y condiciones de trabajo del Torno CNC en la operación de cilindrado. Tecnología en Marcha. Vol.

27, No 2. Pág 32-39

Responsable del Laboratorio SIMTEC, Escuela de Ingeniería en Producción Industrial del Instituto Tecnológico de Costa Rica, Cartago, Costa Rica. Teléfono: (506)2550-9223. Correo electrónico: juhernandez@itcr.ac.cr.

2 PhD, Profesora de la Escuela de Ingeniería en Producción Industrial del Instituto Tecnológico de Costa Rica, Cartago, Costa Rica. Teléfono: (506)2550-921 I. Correo electrónico: mameneses@itcr.ac.cr

$3 \mathrm{PhD}$, Profesor de la Escuela de Ingeniería en Producción Industrial del Instituto Tecnológico de Costa Rica, Cartago, Costa Rica. Teléfono: (506)2550-92 17. Correo electrónico: fpicado@itcr.ac.cr. 


\section{Palabras clave}

Predicción de rugosidad superficial; diseño de experimentos; técnica de superficie de respuesta.

\section{Resumen}

Uno de los principales problemas del maquinado es alcanzar la calidad definida del producto bajo las condiciones de trabajo de que se disponga. La operación de cilindrado en el torno CNC del Laboratorio de Sistemas Integrados de Manufactura (SIMTEC) ha sido estudiada con diferentes condiciones de corte (avance, profundidad de corte) y tipo de material (aluminio 606I, acero 1020) para identificar la combinación y el nivel de factores que mejoran el acabado superficial de una pieza. Utilizando tres técnicas de diseño de experimentos (DOE), a saber, diseño factorial, regresión lineal múltiple y de superficie de respuesta (RSM), se identifica que los factores y niveles que reducen la rugosidad superficial son el, y el material aluminio. Se ha establecido un modelo de regresión de primer orden que puede ser utilizado junto con la gráfica RMS para predecir el valor de la rugosidad promedio.

\section{Key words}

Surface roughness prediction; design of experiments; response surface methodology.

\begin{abstract}
One of the main problems of machining can be described as the achievement of a predefined product quality with given working conditions. The turning operation on Sistemas Integrados de Manufactura (SIMTEC) Laboratory has been studied with diffe-rent cutting conditions (feed, depth of cut, rpm) and material type (Aluminum 606I, Steel 1020) to identify the combination and level factors that improve the surface roughness in a work piece. Using three of Design of Experiments techniques (DOE), such as factorial design, multiple linear regression and response surface methodology (RSM) we identified the factors and levels that reduce the surface roughness and these are the feed rate $=50 \mathrm{~mm} / \mathrm{min}, \mathrm{rpm}=1200$ and aluminum material. A first-order model has been established between the cutting parameters and surface roughness which can be used together with the RMS graph to predict an average roughness value.
\end{abstract}

\section{Introducción}

En manufactura, a la remoción de material y la modificación de la superficie de una pieza de trabajo se le llama operación de maquinado o mecanizado. Esta operación se lleva a cabo con un sistema compuesto de la pieza de trabajo, la herramienta de corte, la máquina herramienta y el operador. En general, los parámetros que influyen en el proceso de corte (variables independientes) son el material y las condiciones de la pieza de trabajo, el filo y el material de la herramienta, las características funcionales de la máquina herramienta, el avance, la velocidad y profundidad del corte, el fluido de corte y las sujeciones de la pieza de trabajo. Entre las variables dependientes se encuentran el tipo de viruta producida, la energía disipada durante el corte, la elevación de la temperatura en la pieza de trabajo, el desgaste y falla de la herramienta y el acabado superficial de la pieza de trabajo (Kalpakjian y Schmid, 2008).
Uno de los indicadores más utilizados para determinar la calidad del producto mecanizado es la rugosidad superficial de la pieza elaborada. La rugosidad se refiere a las desviaciones con respecto a la propia superficie ondulada, causada por la geometría de la herramienta de corte y sus condiciones de desgaste, las condiciones de mecanizado, la microestructura de la pieza de trabajo y las vibraciones en el sistema, entre otros factores (ASM, 1992). En consecuencia, la variable rugosidad depende de los parámetros de la máquina, las propiedades de la herramienta de corte, las propiedades de la pieza que se va a mecanizar y los fenómenos de corte (Benardos y Vosniakos, 2003, Kalpakjian y Schmid, 2008). Al maquinar una pieza, el operador busca alcanzar el objetivo de acabado definido con el mínimo costo y tiempo de elaboración y con las condiciones de trabajo disponibles.

Debido a la complejidad de los fenómenos que componen el proceso de corte, los investigadores 
han propuesto diferentes estrategias para predecir la rugosidad superficial. Benardos y Vosnaikos (2003) presentan una revisión de la literatura sobre los diferentes enfoques desarrollados para la predicción de la rugosidad superficial, mientras que Yusup y otros (20I2), Mukherjee y Ray (2006) presentan una revisión sobre herramientas de optimización de parámetros.

Refiriéndonos al enfoque para la predicción de la rugosidad, los parámetros o factores de mecanizado que influyen sobre la rugosidad superficial son los procesos cinemáticos, el fluido de enfriamiento, la profundidad de corte, la velocidad de corte y de avance $\mathrm{o}$ alimentación y el ángulo de corte (Benardos y Vosniakos, 2003). Los manuales de máquinas-herramientas recomiendan parámetros de mecanizado específicos, pero estos no necesariamente satisfacen la necesidad de mecanizado económico y sus recomendaciones están hechas para un cierto tipo de máquina y bajo condiciones muy específicas que no necesariamente se tienen a disposición en nuestros laboratorios. También la selección de niveles de cada factor se hace tomando como referencia el juicio y la experiencia del operador, pero la realidad es que son muchos los elementos involucrados en este tipo de procesos y no es tan fácil conocer el afecto del ajuste de los niveles de cada uno de los factores sobre la variable respuesta y sobre el resto de los parámetros.

Mukherjee y Ray (2006) presentan una clasificación de modelos y técnicas de optimizacion para procesos de corte de metal. Entre las técnicas de optimización convencionales se encuentra el DOE, que combina el diseño factorial, el análisis de regresión y la metodología de superficie de respuesta para identificar la combinación de factores y niveles que incrementan el rendimiento de la variable respuesta.

Por otra parte, las investigaciones sobre optimización de parámetros de maquinado se pueden clasificar según sean para técnicas convencionales (torneado, fresado, taladrado o rectificado, entre otras) o procesos más modernos de maquinado, como maquinado por descarga eléctrica (EDM) o corte waterjet (AWJ) (Aggarwal y Singh, 2005, Mukherjee y Ray, 2006, Bharathi Raja y Baskar, 201I, Yusup Mohd Zain y Za, 20 I2, Benardos y Vosniakos, 2003).

El objetivo de este trabajo es identificar el material y las condiciones de mecanizado para realizar la operación de cilindrado con el torno CNC del
Laboratorio SIMTEC que asegure un mejor acabado superficial de la pieza que se elabora. Se utilizará el enfoque DOE para la minimización de la rugosidad superficial. Los beneficios de este experimento están relacionados con la identificación del material de referencia adecuado para la realización de los experimentos con objetivos académicos y la identificacion de los parámetros del proceso que permitan obtener la mejor calidad superficial en la operación de cilindrado. Se ha incrementado además el conocimiento general del proceso de torneado mediante esta máquina herramienta en el laboratorio.

\section{Equipo, materiales y método}

El torneado es un proceso que permite mecanizar piezas de forma geométrica en revolución. Considerando la operación de cilindrado de la figura I, la herramienta de corte se ajusta a la profundidad $p$ (que define la cantidad de material por remover) para desplazarse con velocidad $v_{c}$ (velocidad periférica de corte de un punto de contacto de la pieza con la herramienta) mientras la pieza gira. El avance o velocidad de avance $f$ es la di stancia que la herramienta se desplaza horizontalmente por cada revolución de la pieza (rpm).

El diseño de experimentos es un método estadístico que ayuda a establecer qué variables son importantes en un proceso y las condiciones en que deben trabajar para optimizar el proceso (Montgomery, 2002). El DOE tiene muchas aplicaciones en el área de la ingeniería (Carlyle et al., 2000, Ilzarb et al., 2008)

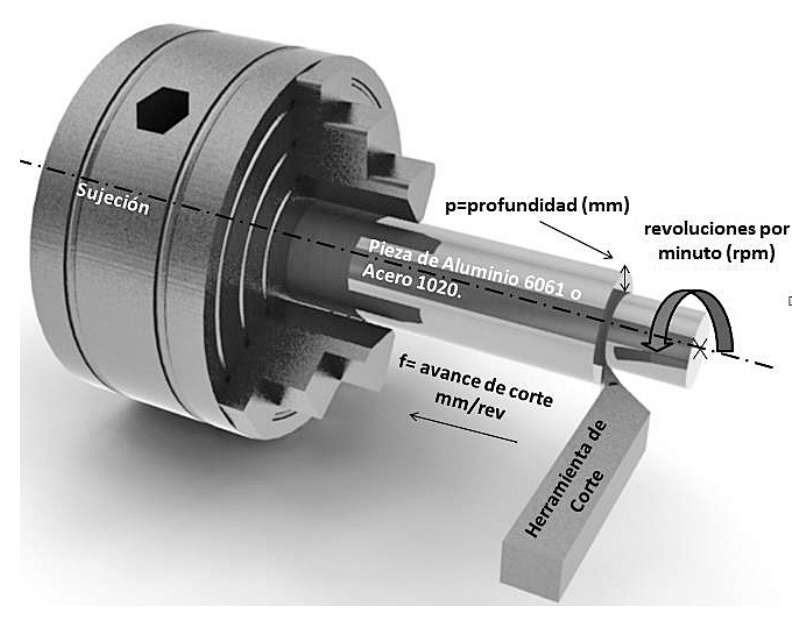

Figura I.Variables en una operación de cilindrado. 
y en específico en la caracterización de técnicas de maquinado (Benardos y Vosniakos, 2003, Aggarwal y Singh, 2005, Mukherjee y Ray 2006). Parte de nuestro objetivo es conocer qué tipo de efecto tienen el material, la profundidad de pasada, las revoluciones por minuto y el avance de corte sobre la rugosidad superficial en la elaboración de operaciones de cilindrado con un torno CNC específico y de acuerdo a las condiciones de trabajo. La pregunta que nos hacemos es jexiste una elección de estas variables que permitá obtener un mejor acabado?

En un estudio con diseño experimental se permite modelar simultáneamente la media del fenómeno estudiado, su variabilidad y observaciones correlacionadas. Las observaciones pueden escribirse mediante el modelo estadístico general:

$y_{i j k z}=\mu+\tau_{i}+\beta_{j}+\gamma_{k}+\omega_{z}+(\tau \beta)_{i j}+\cdots+(\tau \beta \gamma)_{i j k}$

$+\cdots+(\tau \beta \gamma \omega)_{i j k z}+\varepsilon_{i j k z}$

con $i=1,2, \ldots a, j=1,2, \ldots n, z=1,2, \ldots m$, con $a, b, n y$ $m$ el número de niveles de cada factor o variable independiente, y $\varepsilon$ es el término de residuos donde $\varepsilon_{i j k z} \sim N\left(\mu, \sigma^{2}\right)$. El efecto medio general de los factores está señalado con $\mu$ y los términos $\tau_{i} \beta, \gamma_{k^{\prime}} \omega_{z}$ son los efectos específicos de cada factor sobre la respuesta. Las interacciones entre las cuatro variables pueden ser términos dobles, triples y/o cuádruples. El interés ahora consiste en probar las hipótesis de la igualdad de los efectos de cada factor en todos sus niveles y determinar si los factores en cada nivel interaccionan.

\section{Materiales y métodos}

Para los cortes de cilindrado exterior se utilizó un torno CNC marca ACRA, modelo FEL-I440 con control en dos ejes, capacidad de $890 \mathrm{~mm}$ entre centros, rango de velocidad es de 25 a 3000 (rpm), con carrera en su eje $Z$ de $865 \mathrm{~mm}$ y en su eje $X$ de 205 mm. La torreta utilizada es una estándar manual de cuatro posiciones. El motor del torno es de corriente alterna de $5.5 \mathrm{~kW}(7.5 \mathrm{HP})$. La herramienta de corte marca Iscar modelo VNMG 2.5-I-NF es triangular negativo para torneado con dos caras rómbicas de 35 grados; estos insertos son para aplicaciones de semiacabado y acabado con fuerzas de corte bajas debido al borde muy afilado y el desprendimiento de viruta. Se utilizaron 16 cuchillas idénticas para elaborar las 48 piezas, cada 3 piezas se cambió la cuchilla.
Se adquirieron 24 piezas de aluminio 606I y 24 de acero 1020 cuyo diámetro era de 19,05 mm (barra calibrada) y largo variable mayor a $76 \mathrm{~mm}$. Para estandarizar las piezas, estas fueron refrentadas en un torno manual marca JET hasta alcanzar una dimensión de $(76 \pm 1)$ mm. Se limaron los bordes de las piezas para eliminar rebabas de material y evitar falsas mediciones. Para la operación de cilindrado en el torno CNC, se definió un procedimiento para el montaje de cada pieza a fin de asegurar que en cada maquinado se posicionara la pieza en el mismo punto de partida (figura 2).

Se ha diseñado un experimento factorial de cuatro niveles, las variables independientes son el avance $(f)$, la profundidad de corte ( $p$ ), los rpm y el tipo de material; la variable respuesta es el acabado superficial a través de la rugosidad. Se eligen dos niveles para cada factor, uno alto y otro bajo, escogidos con base en la experiencia, porque no se han establecido valores exactos para cada variable (cuadro I).

Se hace uso de fluido de corte en todas las corridas para reducir la fricción y el desgaste y evitar el

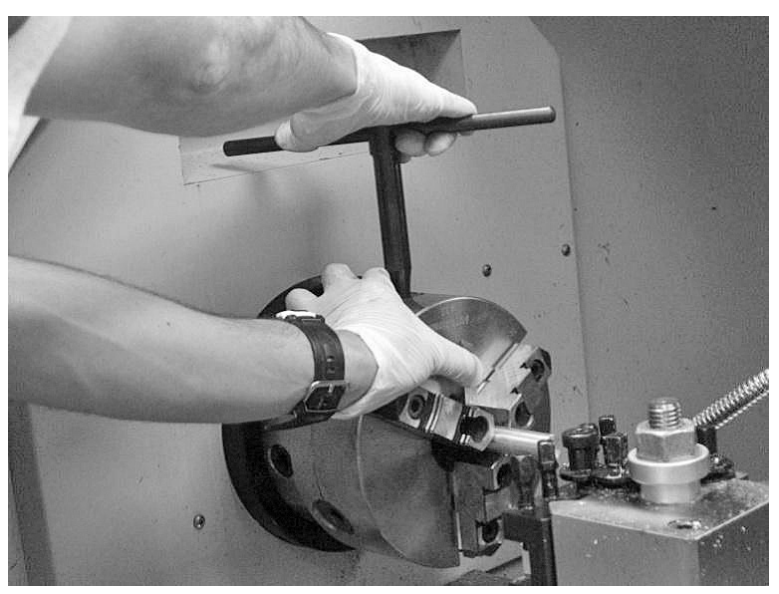

Figura 2. Montaje de la pieza por maquinar en el torno CNC.

Cuadro I. Factores y niveles del experimento.

\begin{tabular}{|c|c|c|c|c|}
\hline Factores & $\begin{array}{c}\text { Profundidad } \\
(\mathrm{mm})\end{array}$ & $\begin{array}{c}\text { Material } \\
(\mathrm{m})\end{array}$ & $\mathrm{rpm}$ & $\begin{array}{c}\text { Avance }(\mathrm{f}) \\
(\mathrm{mm} / \mathrm{min})\end{array}$ \\
\hline \multirow{2}{*}{ niveles } & 2 & aluminio & 900 & 50 \\
\cline { 2 - 5 } & 4 & acero & 1200 & 80 \\
\hline
\end{tabular}


detrimento del acabado superficial. El experimento se lleva a cabo a temperatura constante y utilizando las mismas condiciones para todas las medidas en la herramienta de corte.

Las mediciones para la rugosidad superficial se realizaron con un rugosímetro marca NuLine, modelo TRI00. Se estableció un procedimiento estandarizado para la fijación y medición de cada pieza utilizando un sistema de sujeción con un tope (figura 3) sobre una mesa plana.

El rugosímetro mide la rugosidad promedio $R a$ del valor absoluto de las desviaciones verticales respecto de la superficie nominal. Las mediciones son en micrómetros.

\section{Resultados y discusión}

Se utiliza el software MINITAB 16 para el análisis estadístico del experimento. De acuerdo a los 4 factores se realizó un experimento $2^{4}$ con 3 réplicas para un total de 48 corridas. Se ejecuta el experimento según los resultados de la aleatorización. Cada pieza se identificó con un número en la parte inferior y se marcó según la combinación de los factores. El experimento se hizo en dos días; al ejecutarlo en un torno CNC y realizar un ajuste previo, no se pierden los parámetros al reiniciar la máquina al día siguiente.

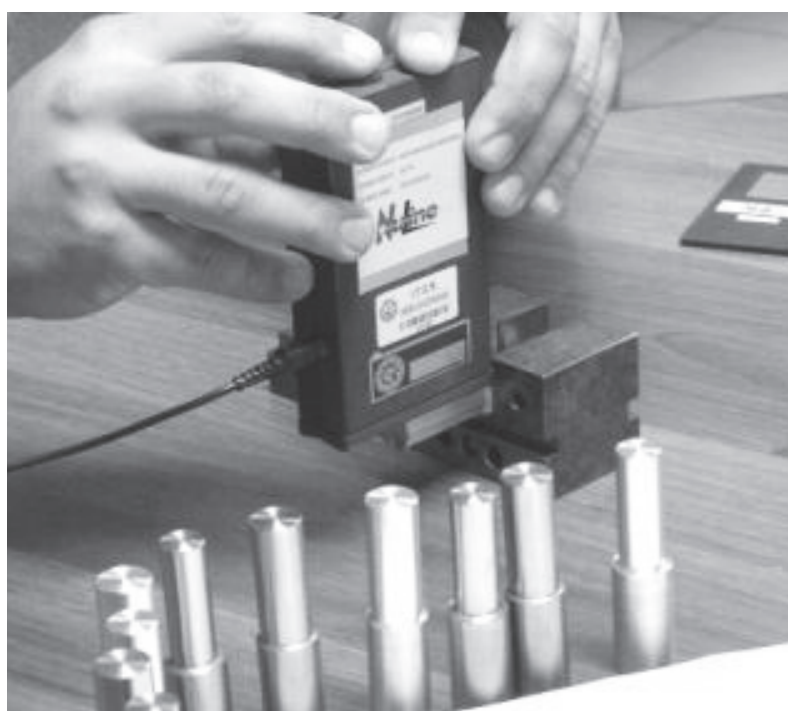

Figura 3. Fijación de la pieza y medición con rugosímetro.
Se estableció un error del primer tipo de $a^{*}=$

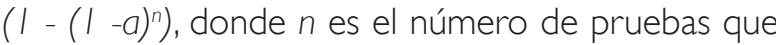
se ejecutan y a es la tasa de falsa alarma nominal total igual 0,05 . El resultado es un modelo reducido en el cual la profundidad no se considera significativa y existe una interacción entre las rpm y el avance ( $f$ ) (cuadro 4).

El modelo resultante con los valores de los coeficientes de regresión es el siguiente:

$Y=1,2975+0,0971 \mathrm{~m}-0,1171 \mathrm{rpm}+0,1237$ $f-0,0950 \mathrm{rpm} * f(2)$

De la figura 4(a) se observa que el material que genera mejor acabado es el aluminio, la profundidad de pasada no es significativa y 4(b) recomienda utilizar avance a $50 \mathrm{~mm} / \mathrm{min}$ y $1200 \mathrm{rpm}$.

El valor de la menor rugosidad obtenida en el experimento es de 0,72 $\mu \mathrm{m}$, generada con las condiciones de avance de $50 \mathrm{~mm} / \mathrm{min}$, rpm $=1200$, y material aluminio. La profundidad, aunque no señalada como significativa con las condiciones de este experimento, fue de $2 \mathrm{~mm}$. De acuerdo con la norma ISO |302, el valor de 0,72 corresponde a un acabado fino porque los límites de esta clasifica-

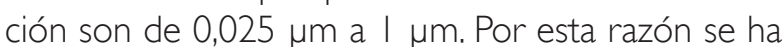
establecido como límite inferior y superior para la optimización de la respuesta una rugosidad mínima de 0,72 um y máxima de I $\mu \mathrm{m}$. La optimización de respuestas identifica la combinación de valores de variables de entrada que en conjunto optimizan la rugosidad. La optimización conjunta debe satisfacer los requisitos de todas las respuestas del conjunto y esto se mide a través de la deseabilidad compuesta (d). El resultado de este método se expone en la figura 5 .

El resultado de optimización de la rugosidad es de I,0546 $\mu \mathrm{m}$, lo que se sale de los límites establecidos y el valor de deseabilidad compuesta es de 0 . Esto indica que la configuración de los niveles de los factores considerada no alcanza los resultados favorables de rugosidad. Se debe continuar con otras pruebas experimentales que analicen no solo otros niveles de factores sino que incluyan también otras variables que no se tomaron en cuenta en este experimento.

La figura 6 muestra un gráfico de contorno con los factores significativos y se indica como ejemplo un punto específico de la variable respuesta, en donde utilizando un avance de 54,9387 y rpm de 1 | 83,53 
Cuadro 4: Resultados del análisis de varianza para rugosidad.

\begin{tabular}{|c|}
\hline Efectos y coeficientes estimados para rugosidad (unidades codificadas) \\
\hline Término Efecto Coef SE CoefT P \\
\hline Constante $1,29750,0356336,420,000$ \\
\hline Material 0,1942 0,097। 0,03563 2,720,009 \\
\hline RPM -0,2342 -0, I I 7। $0,03563-3,290,002$ \\
\hline Avance $0,24750,12370,035633,470,001$ \\
\hline RPM*Avance $-0,1900-0,09500,03563-2,670,01$ I \\
\hline$S=0,24684 \mid$ PRESS $=3,26474$ \\
\hline R-cuad. $=46,52 \%$ R-cuad.(pred.) $=33,36 \%$ R-cuad.(ajustado) $=41,54 \%$ \\
\hline
\end{tabular}

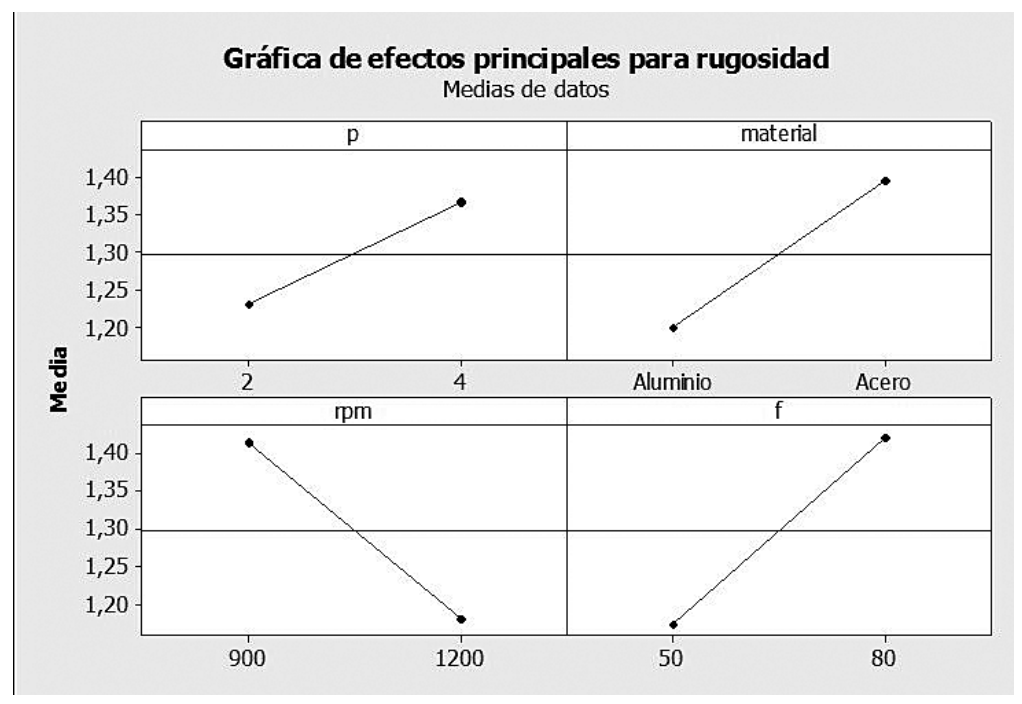

Figura 4. Análisis de efectos principales y de interrelación.

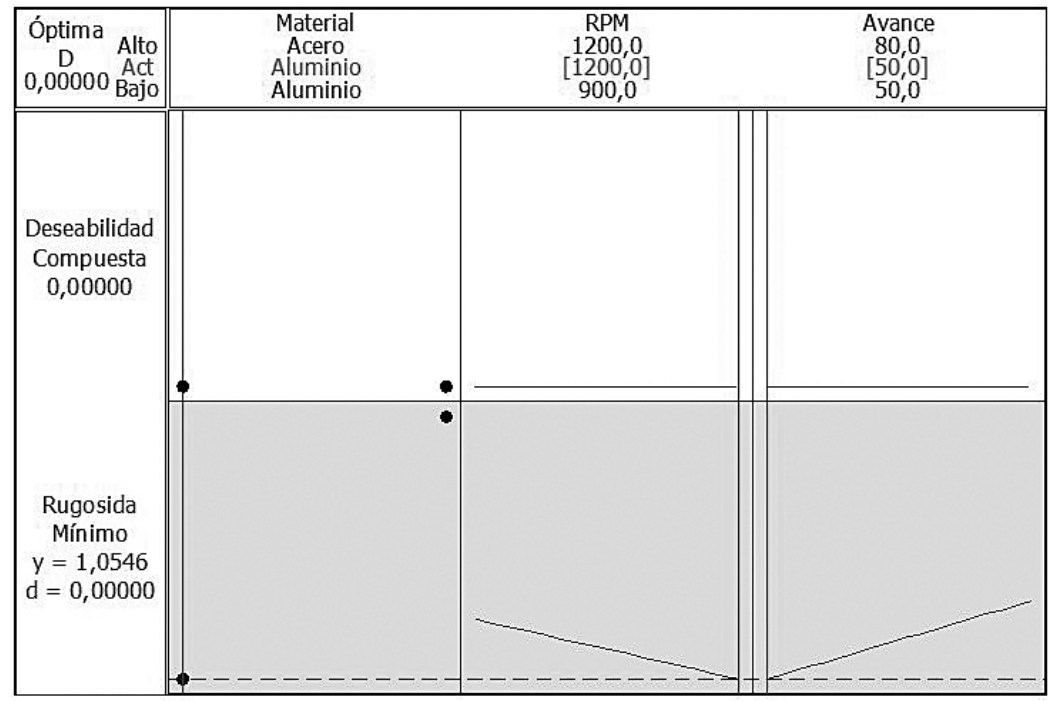

Figura 5. Optimización de la variable de respuesta. 


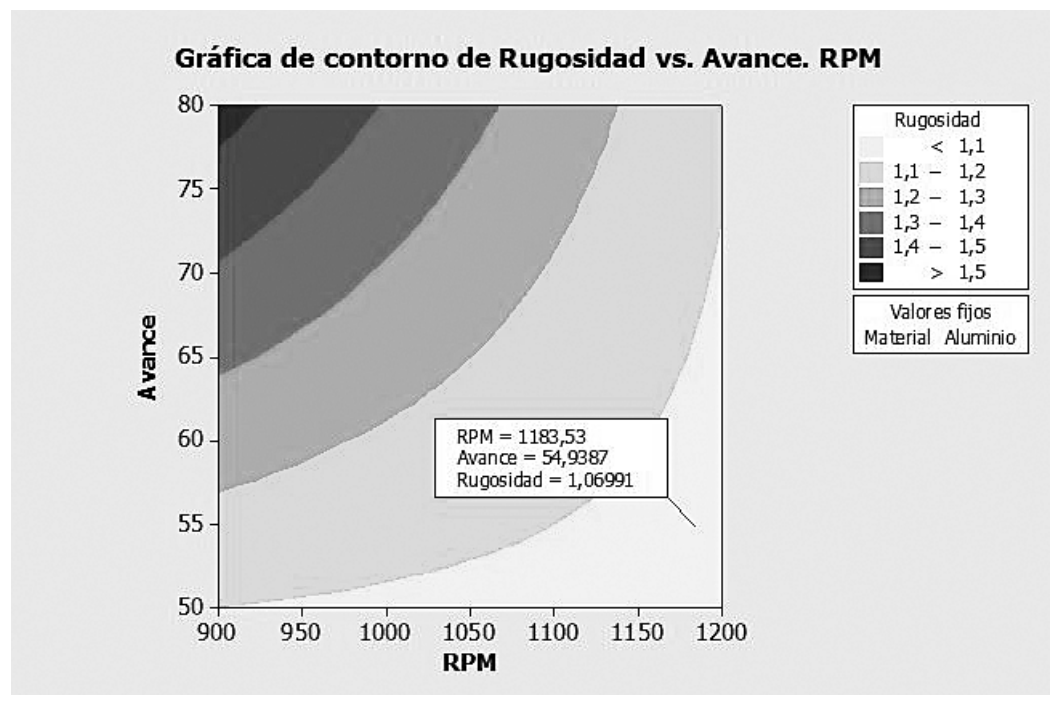

Figura 6: Gráfico de contorno de Rugosidad vrs avance y rpm

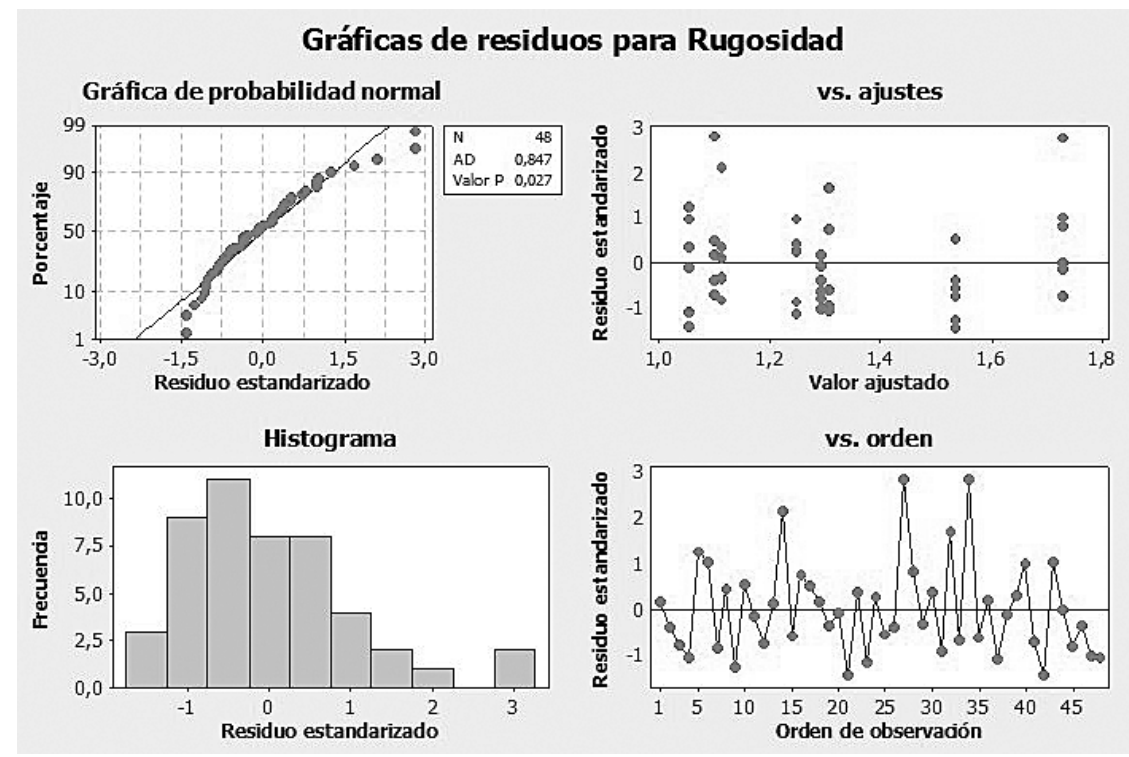

Figura 7. Análisis de residuos del modelo.

en la elaboración con aluminio, se prevé una rugosidad de 1,06991. El gráfico de contorno entonces muestra las zonas donde se podría minimizar la variable de respuesta. Con base en estos resultados, se recomienda realizar pruebas confirmatorias para corroborar las conclusiones experimentales.

La figura 7 muestra el resumen del análisis de los residuos. Los residuos no pasan la prueba de normalidad con Anderson Darling (Valor $P<0,05$ ), que se fundamenta en los valores extremos en las colas de la distribución. Sin embargo, si se utiliza la prueba
Kolgomorov Smirnov, la hipótesis de normalidad es aceptada (Valor $P>0$, I 5). Los residuos son independientes y las varianzas son homogéneas.

\section{Conclusiones}

Se estudió la rugosidad superficial en la operación de cilindrado con el torno CNC del Laboratorio SIMTEC con diferentes condiciones de corte (avance, profundidad de corte, rpm) y tipo de material (aluminio 6061, acero 1020) utilizando un diseño 
factorial $2^{4}$. Las conclusiones de este experimento obtenidas bajo las condiciones especificadas son:

- Los factores significativamente influyentes en la determinación de la rugosidad superficial son el tipo de material, el avance y las rpm.

- Se obtuvo un modelo de regresión de primer orden que puede ser utilizado para estimar el valor de la rugosidad promedio con un margen de confiabilidad del 4I,54\%. Se cumplen los supuestos del modelo estadístico.

- Utilizando el optimizador basado en la función de deseabilidad para minimizar la rugosidad, se obtiene que se logra reducir la variable de respuesta cuando el material es aluminio, $r \mathrm{pm}=$ 1200 y avance $=50$.

- Se debe continuar con la experimentación secuencial e iterativa para ir acercándose al óptimo de la variable de respuesta.

Se recomienda realizar pruebas confirmatorias para corroborar las conclusiones experimentales y realizar estudios teniendo en cuenta otros factores, como por ejemplo, la temperatura y el desgaste de la herramienta, la fuerza y ángulo de corte y el uso de diferentes tipos de lubrificante.

Los resultados orientan al operador en cuanto a la identificación del material de referencia adecuado para realizar experimentos con objetivos académicos e identificar los parámetros del proceso que permitan predecir la mejor calidad superficial en la operación de cilindrado.

\section{Bibliografía}

Aggarwal, A. \& Singh, H. (2005). Optimization of machining techniques. A retrospectiveand literature review. Sadhana 30: 699-7।I.

ASM Handbook, Friction, Lubrication and wear technology, I8, ASM International 1992, pp. 334-335.

Benardos, P. \& Vosniakos, G.C. (2003). Predicting surface roughness in machining: a review. International Journal of Machining Tools \& Manufacture 43: 833-844.

Bharathi Raja, S. \& Baskar, N. (20I I). Particle swarm optimization technique for determining optimal machining parameters of different work piece materials in turning operation. Int J Adv Manuf Technol 54: 445-463.

Carlyle, W., Montgomery, D. \& Runger, G. (2000). Optimization problems and methods in quality control and improvement. Journal of Quality Technology 32(1): | -31.

Ilzarbe, L., Alvarez, M., Viales, E. \& Tanco, M. (2008). Practical Applications of Design of Experiemnts in the Field of Engineering: a bibliographical review. Quality and Reliability Engineering International 24: 417-428.

ISO 1302:2002. Indicación de calidad superficial en la documentación técnica de productos.

Kalpakjian, S. \& Schmid, S. (2008). Manufactura, Ingeniería y Tecnología. Ed. Pearson.

Montgomery, D. (2002). Diseño y análisis de experimentos. Limusa Wiley.

Mukherjee, I. \& Ray, P. (2006). A review of optimization techniques in metal cutting process. Computers \& Industrial Engineering 15-34.

Yusup, N., Mohd Zain, A. \& Za, S. (20/2). Evolutionary techniques in optimizing machining parameters: Review and recent applications (2007-20 II). Expert Systems with Applications 39. 